\title{
Bioética en América Latina: garantizar la vida humana reconociendo su origen primigenio e inaplazable sostenibilidad.
}

Bioethics in Latin America: guaranteeing human life by recognizing its original origin and unavoidable sustainability

\section{Blanca Andrea Ortega Marín ${ }^{1}$ \\ https://orcid.org/oooo-0002-6821-8239 \\ ${ }^{1}$ Universidad Tecnológica de Tulancingo, Hidalgo. México}

\section{PALABRAS CLAVE}

Bioética, Derechos humanos, Desarrollo sostenible, Cambio climático, América Latina.

\section{KEYWORDS}

Bioethics, Human rights, Sustainable development, Climate change, Latin America.

\section{CITAR COMO}

Ortega Marín BA. Bioética en América Latina: garantizar la vida humana reconociendo su origen primigenio e inaplazable sostenibilidad. Rev. cienc. forenses Honduras. 2021; 7(2): 53-63. doi:10.5377/ rcfh.v7i2.13195

\section{AUTOR PARA CORRESPONDENCIA}

Dra. Blanca Andrea Ortega Marín:

blancaortega@utectulancingo.edu.mx

\section{HISTORIA DEL ARTÍCULO}

Recepción: 20 -11- 2021

Aprobación: 8 -12- 2021

\section{DOI}

https://doi.org/10.5377/ rcfh.v7i2.13195

DECLARACIÓN DE RELACIONES $Y$ ACTIVIDADES FINANCIERAS $Y$ CONFLICTOS DE INTERÉS

Ninguna

\section{RESUMEN}

Justificación: la imparable contaminación y uso de los recursos fósiles ha desencadenado innumerables efectos en la naturaleza, la población, la economía; genera migración, pobreza y vulnerabilidad en las poblaciones menos protegidas como las de América Latina. A un siglo de las declaraciones de estudiosos de diversas disciplinas acerca del ámbito de acción de la bioética, sus disertaciones llevan al reconocimiento de que la naturaleza y los seres vivos están relacionados y si se altera su medio ambiente, se arriesga la sobrevivencia. Objetivo: presentar una reflexión sobre la importancia de los recursos naturales en América Latina y su relación con la bioética. Metodología: se realizó una búsqueda de publicaciones de acceso libre en Google Académico, utilizando los términos bioética, cambio climático, pobreza, vulnerabilidad, derechos humanos, desarrollo sostenible y América Latina. Resultados: se seleccionaron 37 artículos comprendidos entre 1970 y 2020 y se agruparon en tres vertientes de interés: los que se centran en la historia de la bioética, los que contenían reflexiones sobre el papel del medio ambiente en la reproducción de la vida, el bienestar, 


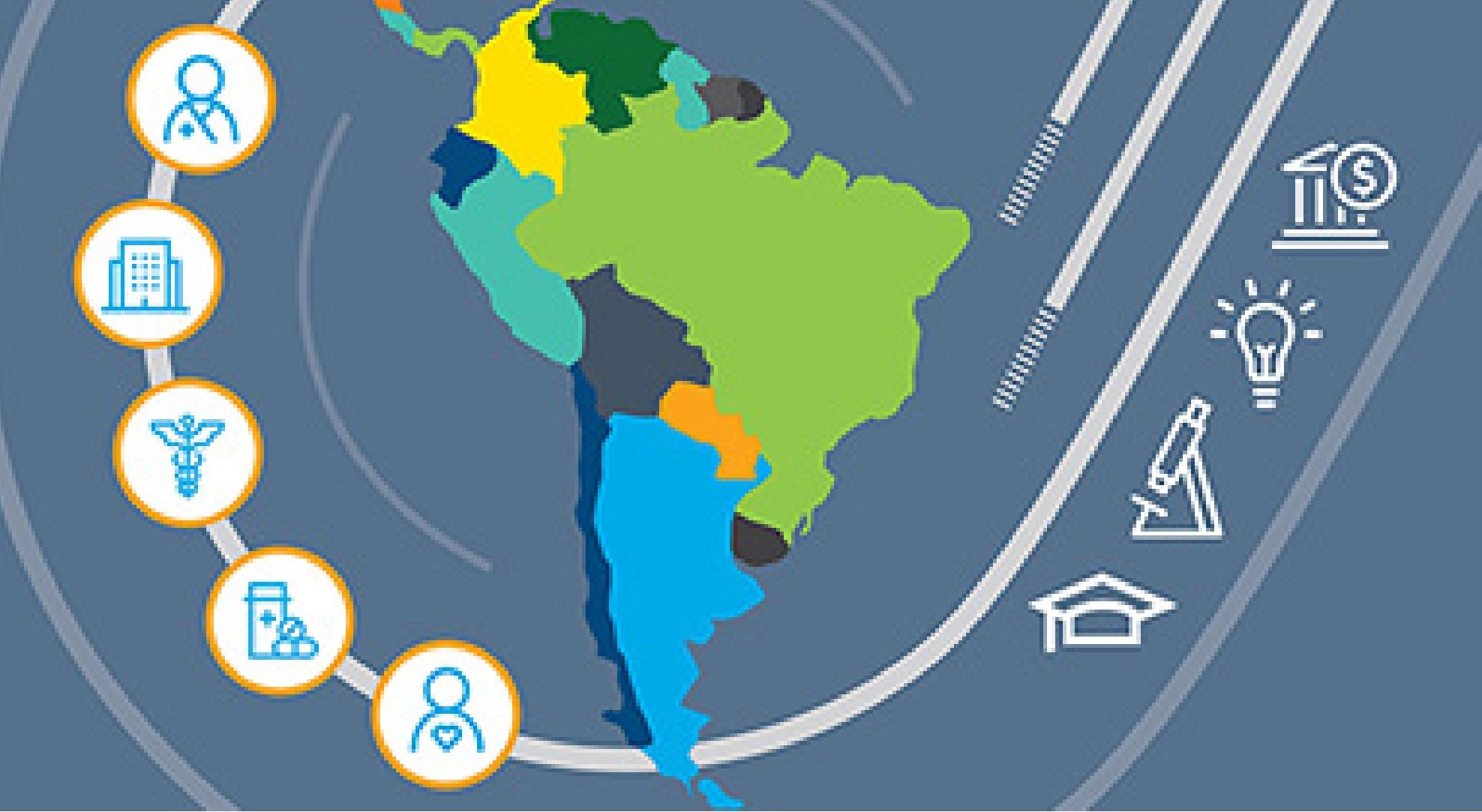

el derecho de las personas a este y los que tomando en consideración el alcance de la bioética; llaman a la responsabilidad individual hacia los recursos naturales y su relación con la realidad latinoamericana de cara al cambio climático. Conclusión: la visión bioética debe ampliarse del enfoque de investigación y su paso de lo médico, a la relación indivisible entre los seres vivos y la naturaleza, los derechos humanos, a una vida sana y el recuento de los retos que vive/sortea cotidianamente la población en América Latina ante las alteraciones ambientales.

\section{ABSTRACT}

Justification: The unstoppable pollution and use of fossil resources has unleashed innumerable effects on nature, the population, and the economy: it generates migration, poverty, and vulnerability in less protected populations such as those of Latin America. A century after the declarations of scholars from various disciplines about the scope of action of bioethics, their dissertations lead to the recognition that nature and living beings are related and if their environment is altered, survival is at risk. Objective: To present a reflection on the importance of natural resources in Latin America and their relationship with bioethics. Methodology: A search was carried out for free access publications in Google Scholar, using the terms bioethics, climate change, poverty, vulnerability, human rights, sustainable development, and Latin America. Results: 37 articles between 1970 and 2020 were selected, they were grouped into three areas of interest: Those that focus on the history of bioethics, those that contained reflections on the role of the environment in the reproduction of life, well-being, the right of people to it and those who, taking into consideration the scope of bioethics, call for individual responsibility towards natural resources and their relationship with the Latin American reality in the face of climate change. Conclusion: The bioethical vision must be broadened from the research approach and its passage from the medical, to the indivisible relationship between living beings and nature, human rights, to a healthy life and the counting of the challenges that the population lives / faces on a daily basis in Latin America in the face of environmental alterations. 


\section{INTRODUCCIÓN}

El 5 de septiembre de 2021 la UNESCO celebró dos décadas de unir la bioética con la ciencia y de estar atentos a que lo científico nunca sobrepase la capacidad crítica ante lo importante ${ }^{1}$. Sumado a esto en noviembre del 2021 se realizó en Glasgow, Reino Unido la COP26 enfocada -entre otros- en la reducción de las emisiones y los efectos de los grados de temperatura planetaria "máxima"2, así como otros fenómenos ambientales como la muerte masiva de abejas en todo el mundo por el uso de pesticidas ${ }^{3}$.

\section{La relación entre la bioética y el} bienestar de las personas, un enfoque que desde la antiguiedad se consideró;

la responsabilidad profesional fue circunscrita a la bioética.

Por otro lado, respecto a la relación entre la bioética y el bienestar de las personas, este es un enfoque que desde la antigüedad fue considerado por el Rey Hammurabi en el Imperio Babilónico en Mesopotamia y entre las importantes consideraciones registradas en el Código Hammurabi (1750 A.C. $)^{4}$, circunscribió la responsabilidad profesioinal a la bioética. Con el paso del tiempo esta conceptualización fue retomada mediante diversas reflexiones sobre las acciones del hombre, la naturaleza y la fauna (1927), la ética de la conservación (1933), la ciencia de la sobrevivencia (1970), como puente hacia el futuro (1971). Bajo estas consideraciones podemos definir la bioética como: el estudio sistemático de la conducta humana en el campo de las ciencias de la vida y del cuidado de la salud, examinada a la luz de los valores y principios morales (1978); incluyendo sus implicaciones antropológicas y éticas, con la finalidad de ver racionalmente aquello que es bueno para el hombre, las futuras generaciones y el ecosistema ${ }^{5}$.

\section{Bioética: su relación con el cambio climático}

El avance económico en países altamente desarrollados ha sido posible por el uso sistemático de los recursos naturales, -particularmente los fósiles-, que han llevado al planeta Tierra al borde del colapso por los imparables niveles de contaminación generados a lo largo de los siglos y el crecimiento poblacional que demanda cada vez más bienes y servicios. Lo anterior propició el incremento de la temperatura planetaria, originando el cambio climático y trayendo consigo diversas alteraciones en el medio ambiente ${ }^{6}$, con consecuencias tales; que gran parte de la población mundial, en general, y en los países latinoamericanos en particular, viven en una situación de vulnerabilidad y carencias ${ }^{7}$.

Los nuevos escenarios del cambio climático imponen a la Bioética una reflexión para que emerja la construcción de un discurso persuasivo orientado a la armonización con la naturaleza

Los nuevos escenarios del cambio climático imponen a la Bioética una reflexión para que emerja la construcción de un discurso persuasivo orientado a la armonización con la naturaleza vista esta como el conjunto de todo lo que forma el universo, que considere al planeta Tierra como un corpus en el que todas sus partes son una unidad y que cualquier alteración lleva a su desintegración. Esto sugiere una apuesta por la conservación y cuidado de los recursos naturales, tarea que debe asumir la bioética; entendida como disciplina reflexiva y crítica sobre las acciones
Volumen 7, N. 2, Año 2021 (C)Autor(es) 
humanas, cuyo paradigma debe ser el concepto de sustentabilidad como medio para lograr el equilibrio y la supervivencia, la promoción de la salud humana de manera concurrente con la preservación del medio ambiente. En este sentido, la bioética asume la responsabilidad con el medio ambiente, como un imperativo colectivo ${ }^{8}$.

\section{Bioética: su relación con los derechos humanos}

La profunda desigualdad que impera en los países latinoamericanos, que conlleva a que una parte importante de la población viva en una situación de mayor vulnerabilidad y carencias; esto orilló a que se formularan, primero, las metas del milenio y en la actualidad los objetivos del desarrollo sustentable, a fin de "alcanzar y/o dotar" de satisfactores y/o servicios a los miles de millones de personas que los requieren ${ }^{9}$. Lo anterior llevó a enriquecer el ámbito de la bioética a partir de la aplicación de las reflexiones más amplias de los expertos y sumarse al llamado mundial al sostenimiento en las ciudades a partir de los recursos naturales de América Latina, unir esfuerzos en torno a esta tarea que, al parecer, adelantó el futuro ${ }^{10}$. En tal sentido, los Derechos Humanos constituyen, básicamente, el punto de referencia para mantener una relación vinculante entre las personas y las naciones-estados, a fin de mantener el respeto jurídico nacional e internacional de la dignidad humana, desde este enfoque los derechos humanos son el mínimo moral, indivisiblemente unidos a la bioética. A partir de la pandemia COVID-19, América Latina mostró dramáticamente la situación de precariedad en la que vive, pese a que los recursos naturales de estos países, son fundamentales para la sobrevivencia mundial, ya que suman arriba del $60 \%$ de la biodiversidad planetaria ${ }^{9}$.

\section{METODOLOGIAA}

Se realizó una búsqueda de publicaciones de libre acceso, disponibles en Google Académico, utilizando los términos bioética, cambio climático, pobreza, vulnerabilidad, derechos humanos, desarrollo sostenible, América Latina y/o Latinoamérica, dando preferencia a las publicaciones de los últimos cinco años y que los estudios realizados abarcaran el periodo comprendido entre 1970-2020.

\section{RESULTADOS}

De los artículos localizados en la búsqueda, se seleccionaron 37, priorizando los que presentaron análisis más amplios a partir del objetivo de la investigación (asimismo a los que incluían el período entre 1970 y 2020). Hay que señalar que todos presentan gran riqueza de contenidos en las dimensiones relativas a la historia de la bioética a lo largo del tiempo, en los continentes, la evolución del concepto de acuerdo con los acontecimientos médicos, filosóficos, económicos y la inclusión de las perspectivas a partir del desarrollo, incorporando la dimensión ambiental, los recursos naturales, el recuento puntual de los eventos que determinaron considerar elementos adicionales de análisis, ampliar y/o enriquecer las disertaciones sobre los temas y visualizando la interdisciplinariedad en su abordaje. En este orden de ideas, es posible agrupar los materiales en tres vertientes de interés.

1. Los que se centran en la historia de la bioética, con diversos planteamientos, desde el desarrollo del concepto a partir de los acontecimientos y lugares donde fue parte del quehacer médico. Posteriormente, en la identificación de la relación entre el ser humano y los recursos naturales, de su importancia para garantizar la sobrevivencia, desde Leopold (1949) hasta nuestros días. Se identificaron también las 
escuelas que se crearon en torno al concepto: la norteamericana, más tarde la europea y en último lugar la Latinoamericana. Todas surgieron en diferentes décadas, con experiencias particulares, y en el tema que nos ocupa, la región latinoamericana en la que se abordó su estudio. Al mismo tiempo las innumerables experiencias económico-sociales en los países se integran dando paso a diversos retos a atender, que llevaron de manera natural a reclamar la acuñación de la Bioética Social, especialmente para los latinoamericanos.

2. Las publicaciones que contienen reflexiones sobre el papel del medio ambiente en la reproducción de la vida (en el concepto más amplio), el bienestar, el derecho de las personas a este, las instancias surgidas en el seno de la Organización de las Naciones Unidas -particularmente en el Programa de las Naciones Unidas para el Medio Ambiente (PNUMA)- y las innumerables reuniones con los representantes de los países miembros que a lo largo de tres décadas -aún continúan "discutiendo las acciones"; mientras el planeta Tierra exige acciones sistemáticas y de largo alcance- hasta los Objetivos del Desarrollo Sostenible 2030 y dentro de la UNESCO; ya dos décadas con el reconocimiento de la bioética como parte de su quehacer ${ }^{8,9,11,12}$.

\section{Los que tomando en consideración el} alcance de la bioética; llaman a la responsabilidad individual hacia los recursos naturales y animales ya que contribuyen a que los humanos se alimenten, lo extrapolan a la realidad de su país y se preguntan ¿por qué en América Latina, que aporta arriba del 60\% de la biodiversidad y hace posible la reproducción de la vida en el planeta Tierra, alrededor del $70 \%$ de su población (420 millones de personas al 2021) vive en el atraso y se han ido sumiendo en la pobreza, en las carencias, en vulnerabilidad y con posibilidades lejanas de salir de ella? ${ }^{7,9}$. Los estudios seleccionados destacan la "tarea asignada" a Latinoamérica de proveer de diversos recursos naturales a varios países del mundo y, desde la década de 1940, considerados tercermundistas, se caracterizó por su atraso económico y tecnológico, surgiendo diversos problemas sociales que, al profundizarse, dieron paso a la pobreza y vulnerabilidad de la mayor parte de su población. De manera paralela expresan el reconocimiento de la naturaleza innata de los recursos naturales, su contribución a la biodiversidad, garantía de soporte de vida y su relación con la biología, aportación particular de los países que integran Latinoamérica. Siguiendo los acontecimientos al 2021 y ante los resultados del desarrollo económico mundial y los efectos de la pandemia COVID-19, su riqueza natural está $\operatorname{amenazada}^{2,7,9}$ y las acciones emprendidas, según los expertos sobre el tema, llegaron tres décadas $\operatorname{tarde}^{20} \mathrm{y}$ en la actualidad los efectos negativos serán imparables y acumulativos mientras se continúe contaminando como hasta ahora ${ }^{3-17}$.

\section{DISCUSIÓN}

Los postulados bioéticos de carácter socioambiental -muestran los materiales revisados- se fueron construyendo y/o evolucionando a lo largo del tiempo, resultado de las reflexiones/disertaciones de los filósofos, académicos, investigadores, estudiosos, de la disciplina desde la perspectiva moral, social, medio ambiental, responsabilidad individual, colectiva, como derecho humano a una vida sana, al bienestar, de forma integral, destacando la relación insustituible de respeto que debe existir entre el hombre y la naturaleza para coexistir armónicamente y hacer posible la sobrevivencia tanto humana como del entorno y sus elementos. El contenido temático es 


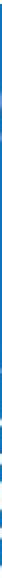

similar y las diferencias detectadas se circunscriben al nivel de profundidad -a partir de la perspectiva y/o interés el autor- ${ }^{18-22}$. Muchos países de los llamados desarrollados crecieron a costa de los recursos naturales de la región latinoamericana, etiquetada como tercemundista a lo largo de las décadas y -poco a poco- cada país que la conforma documentó la situación socioeconómica y ambiental en la que se ha desenvuelto, llevándolos a una creciente desigualdad de oportunidades en todos los ámbitos que integran los derechos humanos, el desarrollo humano, los satisfactores de bienestar -por considerar los conceptos "reconocidos internacionalmente"-. Asimismo, mostrando, ante los efectos del COVID $-19^{5}$, la cruda urgencia de mantener sus recursos naturales: su fuente de vida y reproducción. En este sentido, todos los países largamente beneficiados, tienen una deuda de justicia ambiental, equidad y solidaridad, en especial hacia la población indígena y el sector agrícola -guardianes ancestrales y productores de los alimentos, respectivamente, de sus diversos países (considerando también su riqueza mineral e hidrocarburos) ${ }^{15}$. En este orden de ideas, reconocer la situación del medio ambiente actual -a la vista de todos- y evitar un desastre ambiental largamente anunciado $^{28}$ es una responsabilidad mundial, frente al papel de los recursos naturales en la reproducción humana, que demanda una visión sostenible e interdisciplinaria a la que se sume la población en general.

\section{CONCLUSIONES}

Hay suficiente literatura que aporta un gran conocimiento relacionado con la bioética y su paso de lo médico, a la relación indivisible entre los seres vivos y la naturaleza, los derechos humanos, a una vida sana (incluyendo los componentes correspondientes), el recuento de los retos que vive/sortea cotidianamente la población en América Latina ante las alteraciones ambientales, y todo ello lleva a afirmar que la visión bioética debe ampliarse además delámbito institucional de acción y sumar a la UNESCO que, a dos décadas de incluir a la disciplina entre sus programas, es viable continúe incorporando acciones educativas sobre el tema. Al mismo tiempo, la presión que ejerce la actual coyuntura de la pandemia COVID-19 en la que la misión natural de todos es garantizar la reproducción de la vida en correspondencia a la aportación biodiversa de los países latinoamericanos y sumar en la orientación a la sostenibilidad ${ }^{10,28,29}$ y garantizar también la producción de alimentos ${ }^{33}$. Lograr lo anterior, luego del reconocimiento de las tres décadas en las que se pudieron iniciar las acciones relativas a la sostenibilidad ${ }^{30}$ implica revisar lo que se ha hecho, diseñar nuevas formas de $\operatorname{accion}^{33}$, con una visión 
interdisciplinaria para ampliar la participación ${ }^{32}$, mediante la difusión de estos temas en dos niveles. Es decir, apoyados con políticas públicas relacionadas ${ }^{34}$ con las instituciones educativas y las organizaciones que en Latinoamérica tienen larga experiencia en proyectos rurales ${ }^{31,38}$. Es recomendable ampliar la convocatoria a los estudiantes de las carreras técnicas, licenciatura y posgrado, así como con la población a nivel local, micro regional y/o regional, como la de todos los sectores y gobiernos de los países que se han beneficiado y benefician con los recursos latinoamericanos, para que el impacto de este esfuerzo sea más eficaz. Es posible iniciar con el conocimiento de qué es el medio ambiente, cuáles son sus componentes, cómo se relacionan, por qué requiere ser cuidado, qué tipo de cuidados son recomendables, respectivamente, por indicar los primeros pasos. En un segundo momento, experimentar/vivenciar el derecho a un ambiente sano; iniciando por el acercamiento al concepto, los elementos que lo conforman a fin de que cada persona los reconozca en su entorno, destacar los beneficios intangibles que otorga para facilitar la comprensión personal/colectiva/industrial de las implicaciones si se descuidan, las pérdidas que sobrevienen en el entorno al promover o no acciones comunitarias y/o locales. Ya familiarizados con el contexto y componentes medioambientales, diseñar contenidos específicos sostenibles y a largo plazo, a partir de los requerimientos por nivel de participación, y tipo de población: doméstico, proyectos productivos hortícolas; frutícolas, medidas adaptativas, restauración y/o conservación de los recursos naturales que, de forma paralela, vislumbren la posibilidad de mejorar la calidad de la biodiversidad ${ }^{36}$. Tomar en cuenta las necesidades ciudadanas coadyuvará a despertar el interés en la participación. Asimismo, vislumbrar el diseño conjunto de opciones que se acerquen al disfrute de sus derechos humanos $\mathrm{y}$, considerando las posibilidades, sumar en su desarrollo humano. Estas sencillas iniciativas son posibilidades de interacción en el respeto, $\sin$ violencia $^{35}$ en donde se sientan reconocidos, escuchados aprehendiendo el conocimiento desde su perspectiva que, desde luego, es posible enriquecerla de acuerdo con el alcance que le impriman y sumar en la disminución de su vulnerabilidad. 


\section{REFERENCIAS BIBLIOGRÁFICAS}

\section{1.}

UNESCO. Labor pionera en la UNESCO. [Internet]. París: UNESCO; 2021. [citado 27 agosto 2021]. Disponible en: https://es.unesco.org/news/directorageneral-celebra-dos-decenios-labor-pionera-bioética

\section{2.}

Naciones Unidas. Lo que necesitas saber sobre la Conferencia de las Naciones Unidas sobre el Cambio Climático COP26. [Internet]. NY: ONU; 2021. [citado 27 agosto 2021]. Disponible en:

https://www.unep.org/es/noticias-y-reportajes/ reportajes/lo-que-necesitas-saber-sobre-laconferencia-de-las-naciones-unidas

\section{3.}

Martín-Culma; Arenas-Suárez. Daño colateral en abejas por la exposición a pesticidas. Entramado [Internet]. 2018 [citado 27 agosto 2021];14(1):232240. Disponible en:

http://www.scielo.org.co/pdf/entra/v14n1/1900-3803entra-14-01-232.pdf

\section{4.}

Mark JJ. El Código de Hammurabi. Traducido por Antonio Elduque. En: World History Encyclopedia. [Internet]. Reino Unido: World History Publishing; 2021. Disponible en: https://www.worldhistory.org/ trans/es/1-19882/el-codigo-de-hammurabi/

\section{5.}

González Palomares S. Línea del tiempo sobre la evolución histórica de la bioética en México. Masscience [Internet]. 20 noviembre de 2019. [citado 12 mayo 2021]. Disponible en: https://www. masscience.com/2019/11/20/linea-del-tiempo-sobrela-evolucion-historica-de-la-bioetica-en-mexico/

\section{6.}

Naciones Unidas. Cambio climático y medio ambiente. [Internet]. NY: ONU; 2021. [citado 12 mayo 2021]. Disponible en: https://news.un.org/es/ story/2021/05/1492492

\section{7.}

Naciones Unidas. El número de pobres en la América Latina puede crecer en 35 millones por el coronavirus COVID-19. [Internet]. NY: ONU; 2020. [citado 12 mayo 2021]. Disponible en: https://news.un.org/es/ story/2020/03/1471522 Consulta 26082020.

\section{8.}

Naciones Unidas. Medio Ambiente. Agenda 2030. [Internet]. NY: ONU; 2021. [citado 12 mayo 2021]. Disponible en: https://agenda2030lac.org/es/ organizaciones/pnuma

\section{9.}

Naciones Unidas. América Latina y el Caribe precisan salvaguardar con urgencia la biodiversidad de sus bosques. [Internet]. NY: ONU; 2020. [citado 12 mayo 2021]. Disponible en:

https://news.un.org/es/story/2020/05/1474922

\section{0.}

UNESCO. La UNESCO por las ciudades sostenibles [Internet]. París: UNESCO; 2021. [citado 12 mayo 2021]. Disponible en: https://es.unesco.org/unescofor-sustainable-cities

\section{1.}

Valera L, Marcos A. Capítulo VII. Bioética, ética del medio ambiente y ecología humana: La prudencia como un medio de supervivencia para el ser humano. En: Ortíz AI, Aparicio Meza MJ, Gómes Alvim R. Ecología humana contemporánea en Apuntes y 
visiones en la complejidad del desarrollo. [Internet]. Chile: sn; 2019. [citado 12 mayo 2021]:183-204. Disponible en: https://bioeticalab.uc.cl/wp-content/ uploads/2019/10/Bioética-ética-del-medio-ambientey-ecolog\%C3\%ADa-humana-la-prudencia.pdf

\section{2.}

Pose C. Bioética 50 años más tarde. Revista EIDON. 2020;54(11):1-2. DOI: 10.13184/eidon.54.2020.

\section{3.}

Capó MA, Drane J. Planteamientos bioéticos del medio ambiente. Revista Centro Universitário São Camilo[Internet]. 2014[citado 12 mayo 2021];8(1):4652. Disponible en: http://www.saocamilo-sp.br/pdf/ bioethikos/155560/a3.pdf

\section{4.}

Márquez Mendoza O, Fernández-Carrión MH, Veyta López M, Ruiz Peña So, Guadarrama Guadarrama R. La bioética latinoamericana En: Herreros RuizValdepeñas B, Bandrés Moya F, coordinadores. La bioética en España y Latinoamérica: ¿Hay una bioética iberoamericana? Madrid: Aula Internacional de Biomedicina, Ética y Derechos Humanos FUNDERÉTICA; 2016. [citado 12 mayo 2021]. Disponible en: https://dialnet.unirioja.es/servlet/ articulo? codigo $=6145782$

\section{5.}

Salazar J. Result una visión diferente de la Bioética. Rev Lat Der Méd Med Leg [Internet]. 2001 [citado 12 mayo 2021];6(1):53-56 Disponible en: https:// www.binasss.sa.cr/revistas/rldmml/v5-6n2-1/art8.pdf

\section{6.}

Kopta F. Bioética de la conservación. Rev Pelícano [Internet]. 2020 [citado 12 mayo 2021];6:5-16. Disponible en: http://revistas.bibdigital.uccor.edu.ar/ index.php/pelicano/article/view/1336

\section{7.}

Hernández Ruiz R, Figueroa Aguirre SJ, Ribeiro L. Gestión del aprendizaje bioético sustentable. Rev Aproximacao [Internet]. 2019 [citado 12 mayo 2021];1(1):103-110. Disponible en: https:// revistas.unicentro.br/index.php/aproximacao/article/ view/6253

\section{8.}

Aráujo J, Costa Gómez C. 2030 Agenda and UNESCO's Universal Declaration of Bioethics: basis for social responsibility. [Internet]. Paper presented in 7th International Conference on Ethics Education, Porto, 2019. [citado 12 mayo 2021].

\section{9.}

Burchardt HJ. La crisis actual de América Latina: causas y soluciones Justicia Social. Rev Nuev Soc [Internet]. 2017 [citado 12 mayo 2021]; 267:115128. Disponible en: https://biblat.unam.mx/hevila/ Nuevasociedad/2017/no267/10.pdf

\section{0.}

García H, Limón L. Bioética General. México: Trillas; 2014.

\section{1.}

Valera L. ¿Qué es la ética ambiental desde sus países hasta el futuro? Cuad Bioét [Internet]. 2016 [citado 12 mayo 2021];27:289-292. Disponible en: http:// aebioetica.org/revistas/2016/27/91/289.pdf

\section{2.}

Bonne Annia M, Bonne Gali T. Algunas consideraciones acerca de la Bioética desde una perspectiva global sustentable. Escola Sup Politécnica da Lunda Sul, Saurimo. Revista Eletrônica KulongesaTes (Tecnologia-Educação-Sustentabilidade) [Internet]. 2019 [citado 12 mayo 2021];1(1):129- 
139. Disponible en: http://docplayer.es/208165050Algunas-consideraciones-acerca-de-la-bioeticadesde-una-perspectiva-global-sustentable.html

\section{3.}

Aparicio Payá M. Vulnerabilidad, reconocimiento mutuo y ética ambiental. Bioderecho.es [Internet]. 2018 [citado 12 mayo 2021];8:1-11. Disponible en: https://revistas.um.es/bioderecho/article/view/371521

\section{4.}

Benitez J. Relaciones entre los sistemas de evaluación de impacto ambiental, el desarrollo sustentable y los aspectos bioéticos. Tekhné Rev Fac Ing (Venezuela) [Internet]. 2017 [citado 12 mayo 2021];20(2):25-27. Disponible en: http:/oaji.net/ articles/2019/7118-1560774353.pdf

\section{5.}

Pelluchon C. Elementos para una ética de la vulnerabilidad. Bogotá: Pontificia Universidad Javeriana; 2015.

\section{6.}

Pessini L. Bioética global en tiempos de incertidumbres, perplejidades y esperanzas. [Internet]. Brasil: SalTerrae; 2019. [citado 12 mayo 2021]. Disponible en: https://www.camilianos.org.br/area/img/bioetica global_ebook_FINAL_22fev2019.pdf

\section{7.}

Sánchez M, Antonio J. Reseña de Bioética y medio ambiente De Hottois G, Escobar Triana J, Maldonado C, Cranley Glass K, Couceiro Vidal A, et. al. Rev Colomb Bioét, [Internet]. 2008 [citado 12 mayo 2021]. 3:145-153. Disponible en: https://www.redalyc.org/ pdf/1892/189217505004.pdf

\section{8.}

León Correa FJ, coordinador. Bioética $\mathrm{y}$ medio ambiente. Santiago de Chile Fundación Interuniversitaria Ciencia y Vida. Centro de Bioética UCEN; 2020. [citado 12 mayo 2021].

Disponible en: https://www.pucv.cl/uuaa/site/ docs/20200809/20200809192304/20_bio_tica_y_ medio_ambiente.pdf

\section{9.}

Mora Sánchez AP. Perspectiva de la Bioética en América. Rev Hum Med [Internet]. 2010 [citado 14 abril 2021];10(1). Disponible en: http://scielo. sld.cu/scielo.php?script=sci_arttext\&pid=S1727$81202010000100006 \& \operatorname{lng}=\mathrm{es}$.

\section{0.}

Arellano Hernández A. Cambio Climático y sociedad. Medio Ambiente y Ecología. [Internet]. México: Porrúa; 2014. [citado 12 mayo 2021]. Disponible en: http://ri.uaemex.mx/handle/20.500.11799/59155

\section{1.}

UNESCO. La UNESCO quiere que la educación ambiental sea un componente clave de los planes de estudio para el 2025. [Internet]. París: UNESCO; 2021. [citado 12 mayo 2021]. Disponible en:

https://es.unesco.org/news/unesco-quiere-queeducacion-ambiental-sea-componente-clave-planesestudio-2025 


\section{2.}

Márquez Vargas F. Bioética ambiental en perspectiva latinoamericana. Rev Lat Bioét [Internet]. 2020 [citado 12 mayo 2021];20(2):55-73. Disponible en: https:// dialnet.unirioja.es/servlet/articulo? codigo $=7908439$

\section{3.}

Heinzmann M. Es hora de reflexionar sobre lo que hacemos. Bioética, ambiente y pandemia. Rev Pelícano [Internet]. 2020 [citado 12 mayo 2021]; 6:77-92. Disponible en:

http://revistas.bibdigital.uccor.edu.ar/index.php/ pelicano/article/view/1340

\section{4.}

Márquez F. Bioética ambiental en perspectiva latinoamericana. Rev Latinoam Bioet [Internet]. 2021 [citado 23 de abril de 2021];20(2): 55-73. Disponible en: https://doi.org/10.18359/rlbi.4910

\section{5.}

Rodríguez-Artavia A. Bioética: una estrategia más allá de una política de paz. Rev Colomb Bioét, [Internet].2018 [citado 12 mayo 2021];13(1):117124. Disponible en: https://www.redalyc.org/ jatsRepo/1892/189257376006/189257376006.pdf

\section{6.}

Arce Rojas RS. Aproximaciones para el desarrollo de una bioética forestal a partir del caso peruano. Universidad Ricardo Palma. [Internet].2020 [citado 12 mayo 2021];20(1):107-122. Disponible en: https:// revistas.unimilitar.edu.co/index.php/rlbi/article/ view/4536.

\section{7.}

Ortega-Marín BA, Gutiérrez-Yurrita PJ. Estrategia de alimentación autosostenible regional: recordando el pasado para avanzar a un mejor futuro. Rev Ed Amb, Brasil[Internet].2020 [citado 12 mayo 2021];1(3):8489. 